\title{
Cerebrospinal fluid pressure and glaucoma
}

Diya Yang ${ }^{1,2}$, Ningli Wang ${ }^{1,2}$

${ }^{1}$ Beijing Tongren Eye Center, Beijing Tongren Hospital, Capital Medical University, Beijing Ophthalmology and Visual Sciences Key Laboratory, Beijing, China; ${ }^{2} B e i j i n g$ Institute of Ophthalmology, Beijing Tongren Hospital, Capital Medical University, Beijing, China

The relationship between cerebrospinal fluid pressure (CSF-P) and glaucoma has aroused great interest in recent years. Before this development, elevated intraocular pressure (IOP) had long being considered as the major risk factor for the development and progression of glaucomatous optic nerve damage. ${ }^{1}$ However, numerous studies have shown that there is a relatively large number of patients with typical glaucomatous optic neuropathy (normal-tension glaucoma, NTG) in whom IOP measurements have always been in the normal range $(<21 \mathrm{mmHg}){ }^{2}$ Thus, several questions arise. Why do NTG patients develop glaucoma in the absence of high IOP? Are factors other than IOP contributing to the pathogenesis of NTG?

As early as the 1970 s, Volkov ${ }^{3}$ pointed out that CSF-P could be a counter-pressure to IOP as well as pathogenically associated with glaucomatous optic neuropathy. In a similar manner, Yablonsky et al. ${ }^{4}$ postulated that an abnormally low CSF-P around the optic nerve could be the reason for barotraumatically-induced optic nerve damage in primary open-angle glaucoma (POAG) with normal IOP. However, it was not until recently that retrospective studies by Berdahl et al. ${ }^{5,6}$ and prospective studies by us $^{7,8}$ both found that CSF-P is lower in POAG patients. Moreover, the CSF-P data in POAG with normal IOP from another study done by Jaggi et al. ${ }^{9}$ also reviewed a lower CSF-P.

Although the studies mentioned above providing evidence of low CSF-P in NTG seemingly resolved the better part of the IOP dilemma, the following questions remain:

Correspondence: Ningli Wang, Beijing Tongren Eye Center, Beijing Tongren Hospital, Capital Medical University, Beijing Ophthalmology and Visual Sciences Key Laboratory, No. 1 Dongjiaominxiang Street, Dongcheng District, Beijing, China.

E-mail:wningli@vip.163.com 


\section{Although lumbar CSF-P is low in NTG patients, is their optic nerve subarachnoid space CSF-P low?}

The assessment of CSF-P in clinical studies was based on single lumbar CSF-P measurements, but not on a measurement of the CSF-P in the subarachnoid space (SAS) of the optic nerve. Whether lumbar CSF-P can represent CSF-P in the optic nerve SAS remains unknown. Since a direct measurement of orbital CSF-P is invasive and not acceptable in clinical practice, a 3T-MRI-based imaging technology was developed to measure optic nerve SAS width as a surrogate for optic nerve subarachnoid CSF-P. A study of 39 POAG patients, in which 21 patients had normal pressure (IOP $<21 \mathrm{mmHg}$ ), 18 patients had high pressure (IOP $>21 \mathrm{mmHg}$ ), and 21 subjects comprised the control group, found that optic nerve SAS width was significantly narrower in the normal-pressure group than in the high-pressure group or the control group.10 This result demonstrated that patients with glaucomatous optic nerve damage and normal IOP had an abnormally narrow subarachnoid CSF space around the post-laminar optic nerve suggesting a low CSF-P or, as a corollary, a high trans-laminar cribrosa pressure difference (TLPD).

In addition to the clinical evidence, it remains important to illustrate the relationship among lumbar CSF-P (LCP), brain CSF-P (ICP), and optic nerve subarachnoid CSF-P (ONSP). Eight dogs were used to measure these pressures simultaneously. The results found that the pressures were different with ICP $>$ LCP $>$ ONSP, but correlated with each other $(P<0.001)$. The trans-lamina cribrosa pressure gradient $(T L P G)$ was highest for IOP-ONSP, lower for IOP-LCP, and lowest for IOP-ICP $(\mathrm{P}<0.001)$. During CSF shunting, ICP gradually decreased in a linear fashion together with ONSP ("ICP-dependent zone"). However, when ICP fell below a critical breakpoint, ICP and ONSP became uncoupled and ONSP remained constant despite further ICP decline ("ICP-independent zone"). ${ }^{11}$ We interpret this as a sign of CSF communication arrest between the intracranial and optic nerve SAS. This may be caused by obstructions of either CSF inflow through the optic canal or outflow into the intra-orbital cavity.

\section{Can low CSF-P induce glaucomatous optic neuropathy?}

Yang et al. ${ }^{12}$ conducted an experimental investigation in monkeys to further explore the potential association between an abnormally low CSF-P and glaucomatous optic nerve damage. Four monkeys received a lumbar-peritoneal shunt to reduce CSF-P, while five control monkeys underwent the same procedure but with the shunt remaining closed. Follow-up examinations included regular measurements of IOP and CSF-P, confocal laser scanning tomography of the optic nerve head and retinal nerve fiber layer, and fundus photography. Four eyes of two study-group monkeys developed a continuous loss of retinal nerve fiber layer, and one eye of a third monkey showed a splinter-like disc hemorrhage during the follow-up. The 
other three eyes in the study group and all eyes of the monkeys in the control group remained unchanged. It was concluded that the chronic reduction of CSF-P led to optic nerve damage in some monkeys with experimental and chronic reduction in CSF-P. Whether optic nerve damage corresponded to glaucoma remained unclear.

\section{Can we measure CSF-P for glaucoma patients clinically?}

In the context of an increased TLPD in the pathogenesis of POAG, one may infer that the diagnostic and therapeutic pattern for POAG patients may be changed. Therefore, it seems essential to examine TLPD in POAG patients. However, lumbar puncture for POAG patients is currently controversial and not applicable in a clinical setting. Although some non-invasive ways of measuring ICP have been developed, the accuracy and efficacy of these methods remains dim. More importantly, orbital CSF-P is the key to TLDP, but ethical concerns regarding the invasiveness of the procedure required for direct measurement has stopped us from conducting this type of research. Finding a non-invasive way to measure orbital CSF-P seems essential and applicable.

Based on 3.0T MRI, the Intracranial and Intraocular Pressure Study Group (iCOP) developed an algorithm for a non-invasive measurement of intracranial CSF-P with MRI-assisted optic nerve SAS measuring with intraclass correlation coefficients (ICCS) of $0.87 .{ }^{13}$ Subsequently, an easier formula to estimate CSF-P without measuring optic nerve SAS was established by means of multivariate analysis (CSF-P [mmHg] = $0.44^{\star} \mathrm{BMI}[\mathrm{kg} / \mathrm{m} 2]+0.16^{\star}$ Diastolic Blood Pressure $[\mathrm{mmHg}]-0.18^{\star}$ Age [Years] - 1.91). It confirmed previous investigations that had also reported on associations between higher BMI and higher CSF-P. ${ }^{14,15}$ Applying the formula in an independent test group revealed that the measured CSF-P $(12.6 \pm 4.8 \mathrm{mmHg})$ did not differ significantly $(P=0.29)$ from the calculated CSF-P $(13.3 \pm 3.2 \mathrm{mmHg})$. We then applied this formula to the population-based Beijing Eye Study, which included 3468 individuals, and Central India Eye and Medical Study, which included 4711 individuals; the results showed that in POAG, but not in angle-closure glaucoma, calculated TLPD versus IOP showed a better association with glaucoma presence and amount of glaucomatous optic neuropathy. ${ }^{16,17}$ These results support the notion of the potential role of low CSF-P in the pathogenesis of POAG.

TLPD imbalance may play an important role in the pathogenesis of glaucomatous optic nerve damage. Mathematical model research is vital and must be continued to elucidate the downstream mechanisms of increased TLPD in glaucomatous optic neuropathy. Given that a rational and precise procedure for diagnosing and managing POAG based on TLPD is not currently applicable, obstacles and problems should be conquered and intensive investigations should be continuous. Clinically, IOP reduction still seems the most applicable way of decreasing TLPD and treating POAG patients. It may be of interest to find a way to increase CSF-P non-invasively. 
Moreover, it is also important to estimate the target TLPD, which may be more reasonable than IOP alone.

\section{References}

1. Leske MC, Heijl A, Hussein M, et al. Factors for glaucoma progression and the effect of treatment: the Early Manifest Glaucoma Trial. Arch Ophthalmol 2003;121(1):48-56. Available from: http://archopht. jamanetwork.com/article.aspx?doi=10.1001/archopht.121.1.48 doi: 10.1001/archopht.121.1.48.

2. Drance SM. Some factors in the production of low tension glaucoma. British Journal of Ophthalmology 1972 Mar;56(3):229-242. Available from: http://bjo.bmj.com/cgi/doi/10.1136/bjo.56.3.229 doi: 10.1136/bjo.56.3.229.

3. Volkov VV. Essential element of the glaucomatous process neglected in clinical practice [in Russian. Oftalmol Zh 1976;31:500.

4. Yablonski M, Ritch R, Pokorny KS. Effect of decreased intracranial pressure on optic disc. Invest Ophthalmol Vis Sci 1979;18[Suppl:165

5. Berdahl JP, Allingham RR, Johnson DH. Cerebrospinal fluid pressure is decreased in primary open-angle glaucoma. Ophthalmology 2008;115(5):763-8. Available from: http://linkinghub.elsevier. com/retrieve/pii/S016164200800064X doi: 10.1016/j.ophtha.2008.01.013.

6. Berdahl JP, Fautsch MP, Stinnett SS, Allingham RR. Intracranial pressure in primary open angle glaucoma, normal tension glaucoma, and ocular hypertension: a case-control study. Invest Ophthalmol Vis Sci 2008;49(12):5412-8. Available from: http://iovs.arvojournals.org/article. aspx?doi=10.1167/iovs.08-2228 doi: 10.1167/iovs.08-2228.

7. Ren R, Jonas JB, Tian G, et al. Cerebrospinal fluid pressure in glaucoma. A prospective study. Ophthalmology 2010;117(2):259-66. Available from: http://linkinghub.elsevier.com/retrieve/pii/ S0161642009007337 doi: 10.1016/j.ophtha.2009.06.058.

8. Ren R, Zhang X, Wang N, Li B, Tian G, Jonas JB. Cerebrospinal fluid pressure in ocular hypertension. Acta Ophthalmol:2011-89.

9. Jaggi GP, Miller NR, Flammer J, Weinreb RN, Remonda L, Killer HE. Optic nerve sheath diameter in normal-tension glaucoma patients. Br J Ophthalmol 2011 Mar;11.

10. Wang NL, Xie XB, Yang DY, et al. "Orbital Cerebrospinal Fluid Space in Glaucoma: The Beijing iCOP Study”. Ophthalmology 2012;119(10):2065-2073. Available from: http://linkinghub.elsevier.com/ retrieve/pii/S0161642012003223 doi: 10.1016/j.ophtha.2012.03.054.

11. Hou R, Zhang Z, Yang D, Wang H, Chen W, Li Z, et al. . Intracranial pressure (ICP) and optic nerve subarachnoid space pressure (ONSP) correlation in the optic nerve chamber: the Beijing Intracranial and Intraocular Pressure (iCOP) study. Brain Res. 2016 Mar 15; 1635.

12. Yang D, Fu J, Hou R, Liu K, Jonas JB, Wang H, et al. Optic neuropathy induced by experimentally reduced cerebrospinal fluid pressure in monkeys. Invest Ophthalmol Vis Sci 2014 Apr;55(5):3067-73. Available from: http://iovs.arvojournals.org/article.aspx?doi=10.1167/iovs.13-13657 doi: 10.1167/ iovs.13-13657.

13. Xie X, Zhang X, Fu J. Noninvasive intracranial pressure estimation by orbital subarachnoid space measurement: the Beijing Intracranial and Intraocular Pressure (iCOP) study[J. Critical Care;2013(17).

14. Berdahl JP, Fleischman D, Zaydlarova J, Stinnett S, Allingham RR, et al. Body mass index has a linear relationship with cerebrospinal fluid pressure. Invest Ophthalmol Vis Sci 2012;53(3):1422. Available from: http://iovs.arvojournals.org/article.aspx?doi=10.1167/iovs.11-8220 doi: 10.1167/iovs.118220.

15. Ren R, Wang N, Zhang X, Tian G, Jonas JB. Cerebrospinal fluid pressure correlated with body mass index. Graefes Arch Clin Exp Ophthalmol 2012;250(3):445-446. Available from: http://link.springer. com/10.1007/s00417-011-1746-1 doi: 10.1007/s00417-011-1746-1. 
16. Jonas JB, Wang N, Wang YX, You QS, Xie X, Yang D, Xu L. Body height, estimated cerebrospinal fluid pressure and open-angle glaucoma. The Beijing Eye Study 2011. PloS one. 2014 Jan 29;9(1):e86678.

17. Jonas JB, Nangia V, Wang N, Bhate K, Nangia P, Nangia P, Yang D, Xie X, Panda-Jonas S. Trans-lamina cribrosa pressure difference and open-angle glaucoma. The central India eye and medical study. PloS one. 2013 Dec 6;8(12):e82284. 